Winfried Jung · Bildergespräche 


\section{Winfried Jung}

\section{Bildergespräche}

\section{Zur Funktion von Kunst und Kultur in Theodor Fontanes "L' Adultera"}

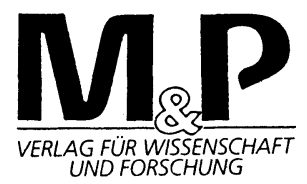


CIP-Titelaufnahme der Deutschen Bibliothek

\section{Jung, Winfried :}

Bildergespräche: zur Funktion von Kunst und Kultur in Theodor Fontanes "L' adultera" / Winfried Jung. Stuttgart: M und P, Verl. für Wiss. und Forschung, 1991 (M\&P) Schriftenreihe für Wissenschaft und Forschung)

Zugl.: Münster (Westfalen), Univ., Diss., 1990

ISBN 978-3-476-45002-9

ISBN 978-3-476-45002-9

ISBN 978-3-476-04160-9 (eBook)

DOI 10.1007/978-3-476-04160-9

Dieses Werk ist einschließlich aller seiner Teile urheberrechtlich geschützt. Jede Verwertung außerhalb der engen Grenzen des Urheberrechtsgesetzes ist ohne Zustimmung des Verlages unzulässig und strafbar. Das gilt insbesondere für Vervielfältigungen, Übersetzungen, Mikroverfilmung und Einspeicherung in elektronischen Systemen.

"M \& P Verlag für Wissenschaft und Forschung ein Verlag der J. B. Metzlerschen Verlagsbuchhandlung und Carl Ernst Poeschel Verlag GmbH in Stuttgart"

(c) 1991 Springer-Verlag GmbH Deutschland Ursprünglich erschienen bei J. B. Metzlersche Verlagsbuchhandlung und Carl Ernst Poeschel Verlag GmbH in Stuttgart 1991 
Ich kann Bildergespräche nicht leiden.

(Theodor Fontane, Cécile) 
Vorbemerkungen

Die vorliegende Arbeit ist die unveränderte Fassung einer im Frühjahr 1990 an der Westfälischen Wilhelms-Universität Münster eingereichten Dissertation, die bei Prof. Dr. Ludwig Völker angefertigt wurde. Inm danke ich für Betreuung und Unterstützung und vor allem für die Geduld, die er mit mir hatte.

Großen Dank schulde ich meinen Freunden, die mir, jeder auf seine Weise, mit Rat und Tat zur Seite gestanden haben.

Ganz besonders danke ich meinen Eltern, die mich in jeder Hinsicht unterstützt haben. Innen sei diese Arbeit gewidmet.

Münster, im März 1991

Winfried Jung 
Inhalt

1. Vorwort 1

2. Bilder im Romanwerk Theodor Fontanes 5

3. Theodor Fontanes "L'Adultera" in der Forschung 21

4. Porträts: Zum Zusammenhang von Bild und

Erzählweise im ersten Kapitel von "L'Adultera"

4.1 Die Bedeutung des ersten Kapitels für die Optik des Lesers

4.2 Die Spannung von Individualität und Typik in der Gattung des Porträts

4.3 Literarisierung und Fiktionalisierung in

Fontanes Figuren-Porträts

4.3.1 Kommerzienrat van der Straaten: individuelles und gesellschaftliches Sprechen im literarischen Zitat

4.3.2 Das Zitat als Selbstdeutung der Figur und als Vorausdeutung des Erzählers

4.3.2.1 Emanuel Geibel: "Rühret nicht daran"

4.3.2.2 Karl Gutzkow: "Uriel Acosta"

4.4 Melanie van der Straaten: literarische 'VorBilder als Ausdruck der Lesererwartungen

5. Bildergespräch I:

Jacopo Tintoretto: Christus und die Ehebrecherin

5.1 Das Bild als "Gegenüberstellung" und "Parallele": Fontanes Wahl des Romantitels

5.2 Die Rezeption Tintorettos im 19. Jahrhundert 
5.3 Tintorettos "L'Adultera". als 'Spiegel' individueller Wünsche und Ängste

5.3.1 Ein "gefährliches Bild": Melanies Deutung von "L’Adultera"

5.3.1.1 Die 'Zweideutigkeit' des Bildes

5.3.1.2 Das Auseinanderfallen von subjektiver Norm und gesellschaftlicher Moral

5.3.2 Das Bild als "Memento mori": Bewältigung und Erzeugung von Angst in van der Straatens Kommentar zu "L’Adultera"

6. Paolo Veronese: Die Hochzeit zu Cana

Das Bild als "Requisit" der Innenraumdarstellung

6.1 Die "sozialindikatorische" Funktion von Bildern

6.2 Das Bild als "Predigt" von der Wand

7. Bildergespräch II:

Kunst, Moral und Politik im Tischgespräch

des 5. Kapitels

7.1 "Heroenkultus": der "Glücksritter" Bismarck

7.2 Madonna und Venus: zur Ambivalenz von Rollenmustern in der Diskussion über "kalte und warme Madonnen"

7.2.1 Murillo: "Immaculata Concepcion" 104

$\begin{array}{lll}\text { 7.2.2 Tizian: Göttin Venus } & 107\end{array}$

7.3 "Wagnerkultus": Wagners Opern als Handlungsmuster in "L’Adultera"

7.3.1 Der "Ritter von Bayreuth": Wagner in seinen Opernfiguren

7.3.2 Ein 'Fensterbild': "Wieland der Schmied" und "Das Kunstwerk der Zukunft" 
7.3.3 Wotans Abschied: "Die Walküre"

7.3.4 "Tristan" und "Tannhäuser"

7.3.4.1 "Himmlische und irdische Liebe"

7.3.4.2 Der Ehebruchs-Mythos

7.3.4.3 Pilgerfahrt

7.3.5 Das Modell der Versöhnung: "Die Meistersinger von Nürnberg"

8. Natur, Kunst und Gesellschaft:

Natur als sentimentale Illusion in "L'Adultera"

8.1 Melanies Porträt: die "Natur der Frau"

8.2 Das Leben im Bild: der Park als "Zaubergarten"

8.3 Das Ende der 'Tragik': die Landpartie

8.3.1 Die Zerstörung des 'Mythos' durch Wiederholung

8.3.2 "Tragische Manieren": die Liebenden im Boot

8.4. Die Beherrschung der Natur:

das Glashaus - ein Mythos des 19. Jahrhunderts 170

$\begin{array}{ll}\text { 8.4.1 Das "künstliche Paradies" } & 170\end{array}$

$\begin{array}{lll}\text { 8.4.2 Der säkularisierte 'Sündenfall' } & 173\end{array}$

8.5 Die Sentimentalisierung der Natur: Goethes

"Wahlverwandtschaften" als Handlungs- und

Reflexionsmodell in "L'Adultera"

8.5.1 Das Palmenzitat als "geflügeltes Wort" 176

8.5.2 Das "Naturgesetzliche" und die Gesetze der Gesellschaft

9. Bildergespräch III:

Der moralische Appell in der zeitgenössischen

Genre- und Historienmalerei 
9.1 Bibelsprüche, Redensarten und Sprichwörter:

Karl Begas: Die Mohrenwäsche

9.2 'Historie' und Gegenwart:

Karl von Piloty: Thusnelda im Triumphzug des Germanicus

9.3 Die Absage an Rollen und 'Vor-Bilder': "Abschied"

10. Bilder von Bestrafung und Belohnung:

Glück, Märchen und Gesellschaft in "L'Adultera"

10.1 Raffael: Das Märchen von Amor und Psyche

10.1.2 Prüfung und Bewährung 221

10.2 Henryk Siemiradzki: Die Fackeln Neros 225

10.3 Schlußtableau: Das verweigerte "Happy end" 229

$11 . \quad$ Bildergespräch IV:

Tintoretto: "L'Adultera". Die Wiederkehr des

Bildes am Schluß des Romans

12. Abbildungen

243

13. Literaturverzeichnis 
1. Vorwort

Das Leben, wie es uns auferlegt ist, ist zu schwer für uns, es bringt uns zuviel Schmerz, Enttäuschung, unlösbare Aufgaben. Um es zu ertragen, können wir Linderungsmittel nicht entbehren. (Es geht nicht ohne Hilfskonstruktionen, hat uns Theodor Fontane gesagt.)'

Was bei Sigmund Freud im 20. Jahrhundert zum Gegenstand der wissenschaftlichen Analyse wird, hat Theodor Fontane in seinen Romanen bereits als ein psychologisches, ästhetisches und gesellschaftliches Problem zugleich formuliert: die Angewiesenheit des Menschen auf die Kunst als Orientierungshilfe in einer allzu komplexen Wirklichkeit. Werke der bildenden Kunst, Bilder unterschiedlichster Art und Herkunft, spielen in Fontanes Romanen häufig als Bezugspunkte seiner Figuren eine wichtige Rolle und vermitteln zwischen der unmittelbaren Begegnung mit der Wirklichkeit und der 'Erkenntnis' von Wirklichkeit. In der vorliegenden Untersuchung soll es nicht primär um Theodor Fontanes spezifisches Verhältnis zur bildenden Kunst gehen, das am Beispiel seines ersten Berliner Gesellschaftsromans "L'Adultera" illustriert würde. Der Roman, dessen Titel sich auf ein tatsächlich existierendes Gemälde bezieht, soll exemplarisch auf Formen und Funktionen des Ästhetischen hin untersucht werden, da in ihm 'Vor-Bilder' aus verschiedenen Bereichen der Kunst die Romanwelt mitkonstituieren und den Handlungsablauf prägen. Um Fontanes Darstellungsabsichten richtig zu erfassen, ist es notwendig, das Ineinandergreifen der verschiedenen Anspielungen und Verweise auf Kunst darzustellen. Dabei orientiere ich mich in Bezug auf Textstrategien, Wahrnehmungen und Erfassungsvorgänge an Überlegungen Wolfgang Isers, die mir im Rahmen der Fragestellung als Beschreibungsmittel des Erzählverfahrens verwendbar erscheinen. ${ }^{2}$ Nachdem in der Erzählforschung lange Zeit der Erzähler als zentraler

Sigmund Freud: Das Unbehagen in der Kultur. Frankfurt/M. 1972, S. 73. Wolfgang Iser: Der Akt des Lesens. München 1976. 
Integrationspunkt der Textmerkmale galt, wird in der Rezeptionsästhetik auch der Leser als implizite Größe des Textes angesehen. An ihm orientieren sich die Darstellungsstrategien des Autors. Hinweise auf Werke der Kunst sind darum Bestandteile der Erzählstruktur und Funktion der bei der Interpretation zu berücksichtigenden Leserlenkungsstrategien. Der dabei entstehende ästhetische Verweisungszusammenhang muß im Laufe des Leseprozesses durch Vorgriffe und Rückbezüge in fortwährender Synthesenbildung realisiert werden. Da dies nur schwer darstellbar ist, soll das zentrale Motiv der im Roman beschriebenen und besprochenen Bilder als Ausgangspunkt der Darstellung dienen und einen Einblick in Fontanes kritisches Erzählverfahren ermöglichen.

Die Tatsache, daß Fontanes Romanfiguren in der Auseinandersetzung mit Bildern immer auch nach ihrer eigenen Identität suchen oder diese durch 'Bilder' bestätigt sehen, führt schließlich zur Frage nach dem Verhältnis von Ich, Wirklichkeit und Kunst in Fontanes Gesellschaftsroman. Die Orientierung an 'Vor-Bildern' aus der Kunst erscheint dabei nicht allein als ein individuelles und psychologisches, sondern vor allem auch als ein gesellschaftliches Problem. Herbert Marcuse hat in seiner kulturphilosophisch-psychoanalytischen Untersuchung "Triebstruktur und Gesellschaft", mit der er Freuds Gedankengänge fortführen wollte, die Verflechtung von Öffentlichem und Privatem als das zentrale Problem unserer Allagserfahrung herausgestellt und die These vertreten, daß die von uns häufig verwendeten psychologischen Begriffe nicht erst in unserem Jahrhundert zu politischen Begriffen geworden sind:

private Verwirrungen spiegeln heute in viel unmittelbarerer Weise die Verwirrungen des Ganzen wider, und die Heilung persőnlicher Störungen hăngt viel direkter als ehedem von der Heilung der Gesamtstörung $a b$. $^{3}$ zu Sigmund Freud. Frankfurt/M. 1979, S. 7. 
Fontane vermittelt eine vergleichbare Zeit- und Gesellschaftskritik, aber auch Einsichten in menschliche Schwächen und Bedürfnisse durch ein Erzählverfahren, dessen kritisches Potential erst durch eine genaue Untersuchung der unterschiedlichen in den Text eingeschriebenen Erzählebenen erkennbar wird. Seine Skepsis gegenüber seiner Zeit und der Möglichkeit der Verwirklichung von Individualität und Glück werden in "L'Adultera" durch unzählige Anspielungen auf Kunst deutlich. Ausgehend von den Bildern, denen die verschiedenen anderen Anspielungen auf Literatur, Theater und Oper zugeordnet sind, soll darum die Frage nach der Bedeutung von Kunst und Kultur in "L'Adultera" zu allgemeineren Aussagen über das Verhältnis von Kunst und Wirklichkeit in Fontanes Gesellschaftsromanen führen. In der Auseinandersetzung mit dem Thema kommen zugleich die zentralen Fragestellungen und Methoden der Fontane-Forschung der letzten 20 Jahre zu Wort. Insofern soll die vorliegende Untersuchung der 'Bilder' in Fontanes "L'Adultera" nicht nur einen Beitrag zur Erfassung der sozialen und der psychologischen Wirklichkeit des späten 19. Jahrhunderts leisten, sondern auch im Sinne einer Standortbestimmung einen exemplarischen Überblick über die Rezeption des Themas 'Kunst und Kultur' in der Fontaneforschung geben. 